\title{
ANÁLISIS Y CONSECUENCIAS DEL MODELO ECONÓMICO LIBERAL EN GUATEMALA
}

\section{ANALYSIS AND CONSEQUENCES OF THE LIBERAL ECONOMIC MODEL IN GUATEMALA}

Referencia del artículo

Martínez Cuestas, M. A. (2021). Análisis y consecuencias del modelo económico liberal en Guatemala. Revista Científica del Sistema de Estudios de Postgrado de la Universidad de San Carlos De Guatemala, 4(1), 61-75. DOI: https://doi.org/10.36958/sep.v4i1.72

\author{
Mtro. Marco Antonio Martínez Cuestas \\ marc027879@gmail.com \\ $0000-0003-1021-3774$ \\ Ingeniero Agrónomo, Maestría en Desarrollo Agropecuario Sustentable \\ Universidad Juarez Autónoma de Tabasco
}

\section{RESUMEN}

La presente investigación se desarrolla mediante la utilización de materiales de información documental, que permitieron realizar un análisis del liberalismo económico en Guatemala, partiendo de los principales exponentes y críticos sociales como: Adam Smith, Karl Marx, Herbert Spencer y otros contemporáneos; generando una línea de tiempo de 60 años, hasta lograr finalizar en el modelo liberal actual; así como los resultados obtenidos a raíz del modelo económico liberal en el país, posteriormente como resultado de los Acuerdos de Paz firmados en el año 1996, dando inicio a una nueva democracia. Además, se presentan los enfoques del liberalismo internacional y la discusión de sus principales características económicas.

En este contexto, el problema de investigación se centra en resolver algunos vacíos del conocimiento que permita comprender las estructuras sociales modernas, lo cual conlleva a preguntar ¿Cuál es la situación actual de los procesos históricos del liberalismo en Guatemala?.

Los indicadores sociales y económicos de Guatemala que describen la evolución del liberalismo en el país permiten generar un estudio comparativo y la propuesta de un proyecto estratégico nacional.

Al finalizar se concluye que el modelo liberal es un fenómeno sustancial que presenta una crisis económica social, debido a la mala utilización de sus preceptos fundamentales, la desigualdad, inequidades económicas, sociales y políticas, presentando una economía tradicional que ignora y empobrece a los individuos de manera material, mental y psíquico; que a pesar de genera extraordinarias ganancias, produce condiciones salariales bajas, con privilegios fiscales particulares y daños al ambiente.

\section{ABSTRACT}

This research is developed through the use of documentary information materials, which allowed an analysis of economic liberalism in Guatemala, starting from the main exponents and social critics such as: Adam Smith, Karl Marx, Herbert Spencer and other contemporaries; generating a 60-year timeline, until achieving completion in the current liberal model; as well as the results obtained as a result of the liberal economic model in the country, later as a result of the peace accords signed in 1996, starting a new democracy. In addition, the approaches to international liberalism and the discussion of its main economic characteristics are presented.

In this context, the research problem focuses on solving some knowledge gaps that allow understanding modern social structures, which leads to the question: What is the current situation of the historical processes of liberalism in Guatemala?.

The social and economic indicators of Guatemala that describe the evolution of liberalism in the country, allow generating a comparative study and the proposal of a national strategic project.

At the end it is concluded that the liberal model is a substantial phenomenon that presents a social economic crisis, due to the misuse of its fundamental precepts, inequality, economic, social and political inequalities, presenting a traditional economy that ignores and impoverishes individuals materially, mentally and psychically; that despite generating extraordinary profits, produces low salary conditions, with particular tax privileges and damage to the environment. 
PALABRAS CLAVE

Modelo, desigualdad, indicadores, economía liberal, oportunidades

\section{INTRODUCCIÓN}

La investigación tiene como finalidad analizar los principales componentes históricos del liberalismo en Guatemala que llevaron al modelo social en la actualidad, con la finalidad de buscar la respuesta a algunas interrogantes del conocimiento que permitan comprender el presente, basado en procesos históricos. Para cumplir con el objetivo se utilizaron diferentes materiales de información documental de carácter científico mediante la aplicación del método deductivo-analítico.

En primera instancia se describe la evolución del liberalismo, desde el pensamiento crítico de Adam Smith y Karl Marx; identificando las características políticas del liberalismo económico desde la perspectiva social, estructural, político y ambiental; permitiendo describir los principales factores del liberalismo que sobresalen en Guatemala, tomando como período de análisis, los últimos 60 años.

De acuerdo con Arriola (2016), Guatemala es un Estado incapaz de promover un impulso acorde a los objetivos de Desarrollo Sostenible -ODS- planteados para el 2030; presentando una contradicción histórica estructural entre el crecimiento económico concentrador de la riqueza y el bienestar social; su modelo económico ha producido desigualdades (Romeo y Ramírez, 2018).

Los indicadores macroeconómicos muestran al país con índices de crecimiento durante el año 2018 de $3.10 \%$ del Producto Interno Bruto -PIB- anual; colocándolo por encima de

\section{KEYWORDS}

Inequality, approaches, model, liberal economy, opportunities

Costa Rica con un índice de $2.7 \%$ y México con 2.0 \% (Expansión, 2018); sin embargo, este crecimiento económico no se ha reflejado en un desarrollo o bienestar para la sociedad en forma equitativa (Romero y Ramírez, 2018).

Aunque el país cuenta con una estabilidad macroeconómica, existe desigualdad y marginalidad en los diferentes sectores sociales; solamente el $1 \%$ de las personas con mayores ingresos en Guatemala, obtienen el equivalente económico del $50 \%$ de las personas con menores ingresos; las grandes empresas, que suponen apenas el $3 \%$ del total de las empresas formales, se llevan el $65 \%$ de las utilidades generadas; por otro lado, las mujeres indígenas rurales reciben ingresos promedios que solo equivalen a una tercera parte de los que ganan los hombres mestizos urbanos (Susanne y Romero, 2019).

\section{MÉTODOS}

Para cumplir con los objetivos de la investigación se utilizó el método deductivo-analítico, enmarcado bajo el esquema del análisis documental, el cual consistió en una serie de procesos sistemáticos que permitió buscar, recapitular, seleccionar, organizar y analizar un conjunto de materiales escritos en forma física y digital, consolidando un documento de información sintética. Este procedimiento permitió el estudio, interpretación y síntesis de diversos documentos que proporcionaron una interpretación sobre los acontecimientos actuales del liberalismo en Guatemala. 


\section{MARCO TEÓRICO}

\section{El Liberalismo y su esencia}

El liberalismo económico con sus cimientos modernos, se inicia durante la segunda mitad del siglo XIX; con una dinámica enfocada en alcanzar el desarrollo económico y la eficiencia en la asignación de los recursos, mediante el establecimiento de un mercado libre donde no existe la intervención del Estado.

Méndez (1998), en su ensayo sobre "el neoliberalismo en México: ¿éxito o fracaso?", manifiesta que:

"Uno de los principales representantes del liberalismo fue Adam Smith; quien en su obra titulada investigación sobre la naturaleza y causas de la riqueza de las naciones, publicada en 1776; presenta los tres principales pilares del liberalismo económico: Libertad personal, propiedad privada, iniciativa y propiedad privada de las empresas".

"El liberalismo económico afirma que la economía de mercado constituye la mejor manera de garantizar el crecimiento económico y de mejorar el nivel de vida de la población de una sociedad dada" (De Vroey, 2009).

Bajo este esquema, sigue mencionando De Vroey (2009) que:

"El liberalismo consiste en un sistema basado en la propiedad privada y en el cual las decisiones de producción se hacen por la iniciativa descentralizada de los agentes económicos, principalmente por las empresas, con el objetivo de obtener beneficios, bajo la señal del sistema de precios y en un contexto de competencia".

Adam Smith defendía el principio básico del egoísmo como motivo fundamental del com- portamiento humano, por tal razón manifestaba que el egoísmo no es un elemento del razonamiento humano, sino un principio emocional. "Como se cree que los conflictos humanos surgen de conductas irracionales, es considerable poder resolverlos a partir de la razón, pero la razón no resuelve conflictos humanos, estos más bien, son resueltos por principios emocionales" (Hevia, 2006); considerando esta premisa se entiende lo complejo que es la sociedad y el estudio de ella.

En su libro el "hombre contra el Estado" Spencer (2014/1851), definió un tratado que se remonta a 1851; en el cual publicó un tratado llamado: "estáticas sociales, o las condiciones esenciales para especificar la felicidad humana". La obra marcó fuertemente el principio fundamental, que la sociedad debía organizarse sobre la base de cooperación voluntaria y no sobre la cooperación forzosa y mucho menos bajo amenaza de ella.

"Los supuestos sobre los cuales se erige el liberalismo económico se circunscriben bajo los principios del liberalismo político de la libertad y la igualdad. Desde la perspectiva económica, la libertad se entiende como un mecanismo que garantiza el pleno disfrute de derechos individuales sustentada en la propiedad privada y en el libre mercado" (Soto, 2002).

Spencer muestra que el liberal primitivo se fundamentaba en el precepto de que el ciudadano pudiera ejercer y regular sus propias actividades tal y como él creyera adecuado, libre de control o supervisión estatal, para reducir sistemáticamente el poder coercitivo del Estado sobre el ciudadano, siempre que fuese posible.

Wallerstein (2015), en su artículo titulado ¿el fin de que modernidad?; muestra la situación del modelo liberal del sufragio, el Estado de bienestar y la identidad nacional que se ofre- 
ció principalmente a las clases peligrosas de los estados centrales, presentando la esperanza de unas reformas graduales pero constantes que prometían los políticos liberales y los tecnócratas, y que significaban finalmente un mejoramiento y una nivelación de las recompensas.

Por otro lado, esa esperanza se ofrecía directamente y también a través de modos más sutiles. Se la ofreció, por ejemplo, en la forma de una teoría de la historia que señalaba como inevitable ese mejoramiento de la condición de vida, bajo el título del in-suprimible camino hacia la libertad humana.

Sin embargo, sigue comentando Wallerstein (2015), que:

"La lucha política-cultural atestiguada entre los siglos XVI y XVIII por sus propios contemporáneos; la doble lucha por la modernidad de la tecnología y la modernidad de la liberación se redefinió retrospectiva y definitivamente en el siglo XIX como una lucha única, centrada en el héroe social del individuo".

Eso representaba la parte medular de la interpretación de una serie de eventos que necesariamente han conducido al presente a través de la historia, que era en parte -de hecho, en su mayor parte- la del proceso de imposición de una geo cultura dominante en la economía del mundo capitalista del siglo XIX.

\section{ANÁLISIS Y DISCUSIÓN}

Méndez (1998), describe que las principales características teóricas del modelo liberal provienen de Hayek y Friedman, destacando las siguientes:

El principio de laisser faire (dejar hacer). El dejar hacer desde la perspectiva de la liber- tad individual, presenta una problemática, a mi parecer, en los principios que orientan a un modelo de economía muy alejados de la comunidad y la colectividad social; muy por el contrario, debería pensarse en una economía integrada, maximizadora de valores monetarios pero también éticos, una economía amigable con el ambiente, con igualdad social, y, a la vez, emocional; bajo un sistema económico de inversión nacional, dejar de pensar que las políticas económicas individualistas no afectan más que al tomador de decisiones, lo cual no es así, debido a la existencia de un entorno espacial con una alta dependencia de esas decisiones.

La libre competencia en el mercado. Una libre competencia de mercado, manifiesta la desigualdad social como resultado del modelo económico, generando un círculo vicioso entre el modelo concentrador que genera desigualdad y la persistente desigualdad que retroalimenta más la concentración económica del país; siendo esta la estrategia de crecimiento sobre la apertura económica en general, la liberación de la cuenta capital, la reducción del Estado (burocracia y funciones), desregulación financiera y tasas de interés libres de acuerdo al mercado, eliminación de toda clase de subsidios sociales, la privatización, la integración regional, seguridad de los derechos de propiedad, entre otras. El problema lo constituye la competitividad espuria en los mercados externos, que generan ganancias extraordinarias al sector exportador en base a bajos salarios, privilegios fiscales y deterioro del ambiente, un precio muy alto que debe pagarse (Susanne y Romero, 2019).

El Estado no debe intervenir en la economía, solo debe garantizar la libre competencia del mercado y estimularla. En este caso, es necesario considerar que el Estado no podría desvincular su participación en los procesos económicos, considerando que este factor y 
la sociedad son componentes asociados y dependientes uno del otro.

Durante el siglo XIX el Estado tuvo que intervenir en varios aspectos de la economía, debido al impacto de la industrialización, para paliar las pésimas condiciones de trabajo de la clase obrera. La reforma inglesa de la década de 1830 y 1840 limitó el empleo de los niños y las horas de trabajo diario y reguló el empleo de las mujeres, al tiempo que nombró inspectores de fábricas; la ley de salud pública de 1848 estableció normas que permitían la intervención del Estado en este campo (Hidalgo, 2001).

La libre circulación de las mercancías, capitales y personas entre los países y, en consecuencia, evitar tanto el proteccionismo como estimular la apertura hacia el exterior del comercio y las nuevas inversiones. No se descarta la idea que la libre circulación de bienes y servicios entre países sea una oportunidad de crecimiento y desarrollo económico, pero también es cierto que el beneficio surge si y solo si, se tienen iguales condiciones competitivas entre los países involucrados; situación que Guatemala no posee; consecuentemente este principio se convierte en una debilidad para el país.

El Estado debe deshacerse de sus empresas y por lo tanto debe venderlas a los empresarios del país y del extranjero. Este es otro factor con el que no concuerdo, debido a que es necesario que el Estado continúe manteniendo y regulando los esquemas que condicionan el funcionamiento del sector empresarial de una manera justa y con la menor brecha de desigualdad posible para todos los colaboradores.

Susanne y Romero (2019), en el documento escrito "entre el suelo y el cielo", manifiestan que existe una desigualdad tan grande en el sector empresario que el $3 \%$ de las grandes empresas se llevan el $65 \%$ del excedente generado, mientras que el $56 \%$ de las microempresas (que lo representan el sector más vulnerable a la crisis económica), accede únicamente al $4 \%$. Este fenómeno presenta un desequilibrio económico en el país y se incrementaría aún más al no existir una regulación del Estado hacia el desarrollo del sector empresarial en forma justa y equitativa, bajo los códigos de ética y laborales existentes.

Prioridad del mercado mundial, más que el mercado interno. Bajo este esquema, como lo menciona Bodemer (1998), los liberales y neoliberales mantienen la concepción del mercado mundial como una nueva era de riqueza y de crecimiento, de oportunidades para nuevos actores, para los que hasta un tiempo atrás eran los perdedores y también para los pequeños países.

Según esta visión, el mercado mundial se presenta como una mejora de oportunidades; pero la discrepancia en este precepto, es que a pesar de existir en Guatemala un crecimiento del comercio, inversiones y finanzas que han ido en aumento, la participación activa directa y los beneficios son de un pequeño sector capitalista que promueve y posee las condiciones competitivas y comparativas para lograrlo; generando una agudizada lucha distributiva a nivel nacional, enfocada en la crisis del modelo económico. Los objetivos básicos de la política liberal son el crecimiento económico, pero con equilibrio financiero, comercial y gubernamental. No se discute que el crecimiento económico sea negativo, pero este principio presenta una fuerte debilidad, debido a que visualiza los factores económicos alejados de la sociedad, lo cual es completamente erróneo. No se puede hablar de equilibrio financiero o comercial, dejando, por un lado, el equilibrio social y ambiental. 
La palabra clave "equilibrio", muestra gran complejidad que es casi imposible su comprensión, posiblemente derivado de lo que afirma Smith y Marx: “...no se puede entender, por qué el término no deriva del razonamiento, sino de la emoción..." (según Aguilera, 2015), manifiesta que factores como el medio ambiente desaparecen de las estructuras mentales y emocionales gracias a que la economía ha enseñado a no poder relacionar ni comprender adecuadamente las cuestiones que se tratan de estudiar como un conjunto, olvidando la dependencia de todo el entorno que nos rodea; enfocándonos únicamente a los modelos matemáticos que pretenden interpretar la economía de los países.

Partiendo de estas premisas, surgen las siguientes interrogantes para Guatemala: ¿Cuáles son los efectos del modelo económico liberal en Guatemala?, ¿Qué factores no permiten un desarrollo económico en el país, a pesar de existir un crecimiento económico en los últimos años?, ¿Los modelos económicos teóricos, solo se limitan a ser simples teorías para el caso de Guatemala?, ¿Las políticas económicas de gobierno no han encajado con las políticas del Estado de Guatemala y sus necesidades sociales?, ¿Es posible que el poder del Estado siempre se ha encontrado en manos equivocadas o con intereses individuales?.

Algunos autores sugieren que la productividad del trabajo puede generar una movilidad social que mejora su bienestar y, que las rentas altas pueden ser generadas por acciones, edificaciones o terrenos que al final producen renta y no solamente por el incremento de la productividad.

Rodríguez (2015a), en el análisis al libro de Thomas Piketty titulado "El capitalismo del siglo XXI", pone de manifiesto un ejemplo muy interesante en cuanto a la productivi- dad; estableciendo que los grandes ejecutivos no son pagados por su productividad, pues muchos de ellos han llevado a la quiebra a sus empresas, tampoco depende de las estrategias empresariales debido a que, por lo general, dominan el mercado.

Efectivamente de manera comparativa y en materia de crecimiento, es posible afirmar que Guatemala ha tenido un comportamiento parecido a los países vecinos, excepto a Costa Rica; los autores, Summers y Atten (2002) atribuyen este factor diferencial principalmente a la orientación de las políticas y la calidad de las leyes, procesos de organizaciones que se vinculan con transacciones de carácter económico según lo afirma una investigación realizada por el Banco Mundial (2001).

Otro factor decisivo, lo representan las instituciones; debido a que el tipo y calidad institucional es determinante para el desarrollo de cualquier país. Un estudio realizado por el Banco Interamericano de Desarrollo (2000), señala que: "el 60 \% de la brecha entre el ingreso de América Latina respecto a los países desarrollados se explica por la calidad institucional".

Estas ideas, llevan a pensar que el desarrollo de Guatemala ha estado marcado por un factor primordial que es la cuestionable calidad de las instituciones del Estado, que la ha distanciado de los países desarrollados del sureste asiático, al igual que el resto de América Latina, a la vez, muestra el potencial de las reformas estructurales, que poco a poco la reinsertan en la senda del desarrollo; como lo afirma Larraín (2006), en su artículo publicado sobre Guatemala: los desafíos del crecimiento. Una aproximación similar realiza Esquivel (2001), quien estudia el "crecimiento económico en Centroamérica" por medio de un enfoque a largo plazo. 
Esquivel (2001) sobre esta línea de investigación, obtiene evidencia sobre el respeto a la ley, la inversión y aspectos externos positivos, que se perfilan como factores de incidencia para mejorar las tasas de desarrollo de los países de Centroamérica, mientras que variables como dificultades en el comercio internacional, los recursos naturales en la estructura productiva del país y el efecto de las catástrofes naturales juegan un papel que impacta negativamente en el crecimiento.

Es necesario hacer la observación que Esquivel, se refiere únicamente al "crecimiento" económico de los países, pero no manifiesta su enfoque al crecimiento económico igualitario, permitiendo, minimizando la brecha de desigualdad existente en los sectores.

Haciendo referencia al ámbito de desarrollo económico es importante considerar el factor de crecimiento demográfico, permitiendo analizar el nacimiento y defunción social. Las investigaciones de Anderson (1983) y otras más actuales como las de Edwards (2000), presentan significativos aportes donde dejan en manifiesto que la relación de la natalidad y mortalidad infantil generan un efecto macroeconómico para cualquier país, ya que se manifiesta directamente en la calidad de vida, mediante la modificación del PIB per capita.

Otro factor de mucha relevancia lo constituye la educación, debido a que el desarrollo de cualquier país se ve correlacionado al nivel educativo de la población, el sector laboral genera más desarrollo económico sostenible, mientras posea una mejor y mayor educación.

Loening (2002) por su parte, "estudia los efectos de la educación en el crecimiento agregado en Guatemala, utilizando datos y técnicas econométricas de series temporales. Su estudio apunta a resolver en parte una carencia en la bibliografía empírica, respecto a la relación entre educación y desarrollo".

"Sus resultados señalan que aumentos en 1.0 $\%$ en la escolaridad promedio del trabajador aumentarían la cantidad producida del trabajador en $0.6 \%$ aproximadamente, aunque los efectos en el crecimiento de largo plazo no son calculables por medio de su modelo, pues dicho efecto depende de la capacidad del país para generar y mantener mayor productividad".

Guatemala, como país, asume el compromiso internacional con los Objetivos del Milenio de alcanzar la universalidad de la cobertura en primaria para el año 2015 , meta que no se logró; por el contrario, la educación sufrió un retroceso (Rodríguez, 2015b).

Este retroceso en educación, según datos de Ministerio de Educación -MINEDUC- (2018), Se ha marcado después de haber llegado en el año 2009 al $98.7 \%$ de cobertura neta en educación primaria, en los últimos gobiernos ha descendido en más de 20 puntos porcentuales, presentando para el 2018 una tasa neta del $77.9 \%$; colocando a Guatemala como la cobertura más baja de Centroamérica.

Para cerrar el círculo de análisis sobre el desarrollo económico de Guatemala, es necesario mencionar la poca elaboración tecnológica imperante en el país, el Banco Mundial (2003) describe que: "es un freno natural al desarrollo del país, dada la importancia de este asunto en las economías modernas".

"De las exportaciones de manufacturas de Guatemala tan sólo 7.5 \% representan productos con alto valor agregado o de alta tecnología. En comparación, las exportaciones de alta tecnología de países de la Organización para la Cooperación y el Desarrollo Económico (OCDE), representan el $23 \%$ de sus 
exportaciones manufactureras totales. Aunque la cifra para la economía guatemalteca no es despreciable respecto al resto de la región; en parte, por el desarrollo de algunas zonas de procesamiento de exportaciones y las maquiladoras". Guatemala no ha podido contar con la tecnología adecuada para ser competitiva en un libre comercio internacional, regulado por el mercado extranjero mediante un modelo económico liberal; únicamente ha sido un instrumento que garantiza la explotación de los recursos internos para beneficio de la comunidad internacional y un sector minoritario nacional.

\section{Medidas económicas liberales en Guate- mala de 1996 a la fecha}

Después del liberalismo, Carlos Collazo (2007), en su artículo publicado "el estado de bienestar" identifica que pasado los años 80's; la ideología de la no participación del Estado paso de moda, pudiendo reconocer el papel importante del Estado para mantener la cohesión social, bajo un esquema de "benefactor social", como lo percibía la sociedad en forma positiva.

Murga (2014), afirma que Guatemala a sufrido una crisis económica debido al modelo de acumulación capitalista centrado en el enriquecimiento desmedido de un sector minoritario, a expensas de la explotación de los recursos naturales, los agronegocios y la construcción de proyectos de infraestructura gigantescos, teniendo serias consecuencias para la nación, como por ejemplo: los desalojo de pobladores, destrucción del medio ambiente, desarticulación de sociedades campesinas, resurgimiento y agudización de conflictos sociales, violación de derechos de comunidades y pueblos indígenas, entre otras.
Muchos factores despóticos han traído consecuencias como el desalojo de comunitarios, daños al ambiente, desarticulación de sociedades campesinas, resurgimiento y agudización de conflictos sociales, violación de derechos humanos, entre otras.

Desde 1996, año crucial para Guatemala, por realizarse la firma de los Acuerdos de Paz, marca una nueva era "democrática" para el país, una democracia basada en un grupo de gobernantes de alta élite que manejan el Estado para ese mismo grupo selecto de individuos; a costa de los efectos negativos producidos a la sociedad y al ambiente, que ha sido promovido y defendido por los gobiernos de turno.

Esto ha traído consigo consecuencias como la ampliación de licencias de explotación minera y petrolera, la política económica de gobierno favorable a la expansión incontrolada de mono cultivos extensivos como; la caña de azúcar y la palma africana, la construcción de represas e hidroeléctricas en diversos puntos del país, etc.

La implementación de un proyecto estratégico nacional con visión neoliberal, tuvo una serie de consecuencias para Guatemala. Ejemplo de ello es la intervención extranjera, principalmente de Estados Unidos de Norte América, teniendo como consecuencias la explotación de la riqueza minera de la nación a cambio de un $1.0 \%$ de regalías, sin considerar el enorme costo ambiental y social. Como lo describe Murga (2014), esto deja como consecuencias los servicios públicos privatizados, siendo uno de los principales la energía eléctrica, la cual trae consigo la necesidad de facilitar las concesiones de los recursos hídricos de Guatemala a empresas privadas, generando consigo el inicio a las políticas energéticas en el gobierno de Oscar Berger (2004-2008). 
Diez departamentos de Guatemala tienen tasas de conexión a la energía eléctrica por debajo de la media nacional de $80.9 \%$. En Alta Verapaz, donde abundan los proyectos hidroeléctricos, solo una tercera parte de los hogares tienen electricidad, es decir, mientras algunos pocos capitalistas nacionales $y$ extranjeros se apropian de los recursos naturales territoriales, la mayoría se queda sin nada (Susanne y Romero, 2019).

Otra consecuencia del proyecto neoliberal es la explotación minera, petrolera, agrícola y los megaproyectos que forman parte del eje de interconexión Física e Integración del proyecto Mesoamérica, que comprende al Sistema de Interconexión Eléctrica para los países de Centroamérica, la Autopista Mesoamericana y los corredores secos Interoceánicos en Centroamérica (El Observador, 2013).

Se inician proyectos de interconexión entre los países vecinos de México, El Salvador y Honduras; presentando un panorama alentador de desarrollo, pero de igual manera a costa de afectar a los más pobres del país; provocando rechazo por esas comunidades afectadas, generando crisis social y guerras civiles en puntos geográficos fronterizos.

Como este caso podrían enumerarse muchos más en Guatemala, pero no es ese el objetivo ni la principal preocupación; aún más preocupante es ver como los gobernantes de turno conocen la gravedad de sus actos, y no hacen lo mínimo para detenerlo. Pudiendo mencionar la administración del Sr. Álvaro Colom, quien adiciona una serie de proyectos hidroeléctricos aprobados y en operación desde 1998, propiedad de grupos multi inversionistas de diversas familias de poder en Guatemala y Honduras; así se continuo con la política privatizadora del sector eléctrico durante la administración del presidente Otto Pérez Molina.
Las concesiones mineras otorgadas a partir de 1997 también colocan al país en manos del gran capital; por ejemplo: en el año 2005 el $10 \%$ del territorio nacional estaba cubierto por permisos mineros, la mayoría de los cuales está en manos extranjeras. Esto, lamentablemente, trae un costo ambiental y social, ¿a cambio de qué?... de un insignificante $1.0 \%$ de regalías para el Estado, las empresas transnacionales provocan la pérdida de acceso a la tierra por las comunidades afectadas, la contaminación del agua y una enorme conflictividad y el caos social imperante; todo debido al modelo económico de libre mercado que propicia el liberalismo.

Si bien todos estos proyectos han contribuido a lograr un crecimiento económico nacional, no contribuyen a un desarrollo económico sostenible, debido a existir una mayor brecha entre ricos y pobres, provocando mayor desigualdad y por lo tanto inconformidad. Como afirma Thomas Piketty en su libro titulado "El capitalismo del siglo XXI" publicado en el año 2013, "cuando más desigualdad existe en una sociedad, mayor es el malestar, generando al final una inestabilidad política poniendo en riesgo el sistema democrático". Proyecto estratégico nacional.

A partir de la firma de los Acuerdos de Paz, se generaron muchas expectativas sobre el efecto que ese suceso, definitivamente marcaría un cambio sustancial para el desarrollo social y económico de Guatemala; considerando temas como la distribución democrática de la tierra, sostenibilidad de los recursos naturales, sistemas y mecanismos de crédito, procesamiento y comercialización, legislación agraria, seguridad jurídica y social, relaciones laborales justas y equitativas, asistencia técnica y capacitación, entre otros; lo cual si fue plasmado y declarado plenamente en los acuerdos firmados; sin embargo, nunca se ha podido llevar a la realidad o ha exis- 
tido poca voluntad política de los gobiernos en turno para poder realizarse.

Guatemala desde hace muchos años, ha presentado un estado fallido en materia de desarrollo social; por ser ineficaz, teniendo sólo un control nominal sobre su territorio y no poder está de más cumplir sus leyes; considerando el incremento de las tasas de criminalidad a un ritmo superior al $12 \%$, superando a ciudades como Detroit, en EEUU; Rio de Janeiro, Brasil y Bogotá, Colombia (Matute y García, 2007).

Por otra parte, presenta índices altos de corrupción, según datos de la Fundación para el Desarrollo de Guatemala -FUNDESA- durante el año 2018, Guatemala ocupó el lugar 144 del ranking de 180 países; presenta una burocracia impenetrable, ineficacia judicial, y a la interferencia militar en la política; un Estado en el cual el principal protagonista inquisidor que atenta en contra del bienestar común es el gobierno, porque las demandas políticas, sociales y económicas, que prevalecen desde el Conflicto Armado Interno, y no han sido resueltas satisfactoriamente por el Estado de Guatemala.

Surgen nuevamente dos preguntas fundamentales como sociedad: ¿tenemos el sistema de gobierno que realmente merecemos? o, por otro lado, ¿será que el sistema gobierno-estado, no permite tener un desarrollo positivo y real para la sociedad guatemalteca? Son preguntas de mucho interés y profundo análisis para considerar en la implementación de un verdadero proyecto estratégico nacional que reactive el desarrollo social y económico del país.

La firma de los Acuerdos de Paz firme y duradera, presentaba un fondo dicotómico; por un lado, la negociación para dar fin a la guerra interna, mientras que, en contraparte un grupo elite del país promovía una ambiciosa campaña por la inversión energética, minera y petrolera en el país.

Considerando la privatización de las empresas estatales y servicios públicos, la desregularización de los mercados, la liberalización del comercio internacional, la eliminación de las barreras a las inversiones extranjeras directas y la protección a la propiedad privada; el consenso de Estados Unidos de América se ajustaba más a sus propios intereses, que los plasmados en los Acuerdos de Paz. Estos, según pudo establecerse posteriormente, eran sólo un requisito para consolidar el modelo económico neoliberal, produciendo una desigualdad mayor entre las esferas sociales, llevando a una crisis económica.

Con las iniciativas de privatización de los servicios públicos, liberación del comercio internacional y protección a la propiedad privada; se impulsa un equivocado modelo liberal de economía en Guatemala, enfocada en que las grandes empresas nuevamente obtengan todas las garantías posibles para sus asuntos, eliminando cualquier obstáculo legal, social o económico que obstaculizará el libre funcionamiento empresarial, bajo el esquema de "Tratado de Libre Comercio" entre Estados Unidos, Centro América y República Dominicana y el famoso plan Puebla Panamá, relegando al Estado al plano de "gestor" de buenas inversiones; reduciéndolo en una simple oficina de fomento de inversión (Murga, 2014).Suanes y Roca (2015), afirman que "la importante afluencia de la inversión extranjera directa dirigida a las economías en desarrollo o emergentes, ha motivado un considerable número de trabajos que intentan explicar su impacto sobre el crecimiento económico y sus determinantes bases del desarrollo".

Esta aseveración no es del todo válida, por dos razones fundamentales; primero, la inversión extranjera ha decaído en los últimos 
cinco años, como lo demuestra el Banco de Guatemala (2019); y en segundo lugar, a pesar de generar fuente de trabajo, la remuneración para la clase trabajadora no es significativa ni representativa, manteniendo siempre una alta desigualdad salarial, reflejada en el poco o nulo desarrollo económico, centralizando el capital en unos pocos; manteniendo a los sectores pobres igualmente pobres, satisfaciendo únicamente las necesidades básicas.

Este fenómeno se puede apreciar en el índice de crecimiento del PIB el cual, muestra una tasa de incremento significativo, una vez descontada la inflación, los salarios han perdido poder adquisitivo, es decir, el crecimiento económico se ha distribuido de forma desigual entre las partes capital y trabajo, provocando una baja en los salarios reales (Banco de Guatemala, 2017).

Hablar de crecimiento económico no significa hablar de desarrollo, igualdad o equidad económica, eso es evidente. Un ejemplo lo constituye el departamento de Petén, ubicado al Norte de Guatemala, que posee diversas características comparativas y competitivas que han permitido el desarrollo del sector agrícola, por poseer un estatus fitosanitario libre de mosca del Mediterráneo declarado por Estados Unidos y México, por tener una ubicación estrategia en puntos fronterizos con México y Belice, por las condiciones agroclimáticas, etc.

Lo que ha permitido exportar durante el año 2018 más de 20,000 toneladas métricas de papaya para Estados Unidos, según datos estadísticos del Programa Integral de Protección Ambiental y Agrícola (PIPAA, 2019). No obstante, el $90 \%$ de estas empresas son extranjeras, que generan inversión, empleo y "manteniendo una economía", con un desarrollo regional, pero con una alta tasa de desigualdad entre los actores involucrados en tan exitosos logros para el país, sin alcanzar un desarrollo en el departamento.

Es posible y fundamental que sobre esas bases las clases empresarial y política en el poder serán los protagonistas de definir los ejes del "modelo económico" que hará de Guatemala un Estado neoliberal de fuertes rasgos coloniales, considerando que durante la colonia, toda la riqueza que se obtenía del país beneficia en gran medida a la Corona española y en menor grado a la clase criolla; con este modelo económico gran parte de esa riqueza beneficia a las empresas extranjeras y en menor grado a la sociedad local. Tenemos, pues, un modelo económico que, en condiciones históricas distintas muestran objetivos similares.

\section{CONCLUSIÓNES}

Considerando los postulados del modelo liberal y en el afán de buscar el crecimiento económico de los países; muestra debilidades en la conformación del desarrollo económico sostenible en Guatemala; acentuando mayores brechas de desigualdad, minimizando la creación de empleos y desfavoreciendo las condiciones de vida para el país.

En los años 50's el Producto Interno Bruto (PIB) per cápita de Guatemala, representaba el $18 \%$ del de Estados Unidos de Norte América, mientras que para el año 2018 esa cifra descendió por debajo de un $10 \%$. A nivel mundial se ha tenido un dominio liberal occidental, marcado después de la Segunda Guerra Mundial, principalmente por Estados Unidos de América; con una visión de Estado, con los ojos enfocados en el control político, económico y social con un fondo muy apegado a sus intereses; principalmente de tipo económico. 
A partir del análisis realizado, se concluye que el modelo liberal imperativo en Guatemala, presenta diversos factores enmarcados en la desigualdad socioeconómica, presentando un modelo basado en la apertura comercial y desregularización financiera, generando extraordinarias ganancias, pero a condiciones de salarios bajos, privilegios fiscales y daños al ambiente. Siendo necesario que el Estado mantenga y regule los esquemás que condicionan el funcionamiento del sector empresarial de una manera justa y con la menor brecha de desigualdad posible para todos los involucrados.

La poca inversión en educación, convenios y tratados de libre comercio únicamente beneficia a un sector pequeño del país, por tener las capacidades de afrontar los retos internacionales bajo las condiciones imperantes de los países extranjeros; manteniendo la concepción del mercado mundial como una nueva era de riqueza y de crecimiento, de oportunidades para nuevos actores, para los que hasta un tiempo atrás eran los perdedores y también para los pequeños países, generando una fuerte lucha distributiva a nivel nacional, enfocada en el modelo económico.

Por otro lado, Guatemala bajo el esquema liberal ha perdido la autonomía en la toma de decisiones, liberando las entradas para la inversión extranjera convirtiéndose únicamente en el moderador para las transacciones económicas, sin tener mayor papel en el desarrollo del país, permitiendo una economía muy alejada de la comunidad y la sociedad; enfocada únicamente en crecimiento de valores monetarios, sin considerar los éticos, morales, ambientales, racional y emocional.

Con todo esto, puede definirse que el modelo económico de Guatemala ha sido el resultado de los sistemas de gobierno fallidos, inconscientes, manipuladores y al servicio de grupos de poder. Con una sociedad su- bordinada, manipulada y con poca identidad social de las masas populares y más necesitadas, con las mismas características de los gobernantes de turno, pensando en el bien individual, olvidándose de la prevalencia del bien colectivo; dominados por gobiernos que conocen las carencias del pueblo, pero consideran que es necesario que continúen de esa manera; lo cual les permite comprar sus emociones y no la razón, considerando la premisa que el "ser humano es más emocional que racional".

Todas estas conclusiones conllevan, a la realización de un análisis de mayor envergadura e importancia, de cómo impulsar el crecimiento económico en el país de manera ambientalmente sostenible y socialmente inclusive.

\section{REFERENCIAS}

Aguilera, F., (2015). Economía y naturaleza humana, volviendo a Smith y Marx. Revista Latinoamericana -Polis-. Ciencias Sociales: desafíos y perspectivas. ISSN: 07186568. Editor Centro de Investigación Sociedad y Políticas Públicas (CISPO). Universidad de la Laguna, Tenerife, España. pp. 01-18. https://doi.org/10.4067/ S0718-65682015000200018

Anderson, K. (1983), "The Determination of Fertility, Schooling and Child Survival in Guatemala", International Economic Review, vol. 24 (3), pp. 567-589. https://doi.org/10.2307/2648787

Arriola, G. (2016). Más allá del conflicto, LUCHAS POR EL BIENESTAR. Informe Nacional de Desarrollo Humano -PNUD2015/2016. pp. 01-404. Recuperado en: http://desarrollohumano.org.gt/ wp-content/uploads/2016/04/INDH16_ Resumen_ejecutivo_digital_FINAL.pdf 
Banco de Guatemala (2017). "Guatemala en Cifras". Departamento de Estadísticas Macroeconómicas. Pp. 01-68 Recuperado en: http://www.banguat.gob.gt/Publica/guatemala_en_cifras_2017.pdf

Banco de Guatemala (2018). "Guatemala en Cifras". Departamento de Estadísticas Macroeconómicas. 68 pp. Recuperado en: http://www.banguat.gob.gt/Publica/guatemala_en_cifras_2018.pdf

Banco de Guatemala (2019). Estadísticas Macroeconómicas. Sector Externo. Inversión Extranjera Directa -IED-. Gráficas de Flujo -IDE. Recuperado en: https: / / www. banguat.gob.gt/inc/main.asp?i$\mathrm{d}=111731$ \& aud $=1$ \&lang $=1$

Banco Mundial (2001), World Development Report 2001/2002, Washington, Banco Mundial.

Bodemer, K. (1998). La globalización. Un concepto y sus problemas. Investigador del Instituto de Estudios Iberoamericanos de Hamburgo. Nueva sociedad, No. 156. Julio-agosto, pp.54-71.

Britnell, G. E. (1951). "Problems of Economic and Social Change in Guatemala", The Canadian Journal of Economics and Political Science, vol. 17(4), pp. 468-481. https: / / doi.org/10.2307/137902

Collazo, C. (2007). El Estado de bienestar. Enfoque XIX, Universidad Adventista del Plata. Paraguay 551, 3100 Paraná. 1-2: pp. 45-54.

De Vroey, M. (2009). El liberalismo económico y la crisis. Lecturas de Economía, (70), 11-38.

Edwards, S. (2000), "Situación macroeconómica en Guatemala: Evaluación y recomendaciones de política monetaria y cambiaria", Banco de Guatemala.

El Observador, (2013). Estudios Estratégicos para la Democracia. Análisis alternativo sobre política y economía. Modelo de acumulación y competitividad. Años 7 y 8, No. 38-39. Colonia Bran, Ciudad de Guatemala. Recuperado en: http:// www. albedrio.org/htm/documentos/ ElObservadorNos38-39diciembre2012abril2013.pdf

Esquivel, G. (2001), "Economic Growth in Central America: A Long Run Perspective", F. Larraín (comp.), Economic Development in Central America, vol. 1: Growth and Internationalization, Harvard University Press.

Expansión (2018). Copyright Datosmacro. com. Producto Interior Bruto. Recuperado en: https://datosmacro.expansion.com/pib

FUNDESA, (2019). Percepción de corrupción. Índice de percepción de la corrupción-IPC- Fundación para el Desarrollo de Guatemala. Recuperado en: https: / / www. fundesa.org.gt/indices-y-evaluaciones-de-pais/indices-internacionales/percepcion-de-corrupcion

Hayek,A.V. (1982). Liberalismo.pp.122-149. Recuperado en: https://repositorio.uc.cl/bitstream/handle/11534/11047/000353390. pdf

Heston, A., Summers R. y Atten, B. (2002), "Penn World Table Version 6.1", Center for International Comparisons at the University of Pennsylvania (CICUP), octubre.

Hevia, A. E. (2006). Desarrollo humano y ética para la sustentabilidad. Universidad Bolivariana. Programa de Naciones Unidas Para el Medio Ambiente -PNUMA-, Oficina Regional Para América Latina y el Caribe. México y Santiago de Chile, Eg. Enriques Leff. ISBN 968-7913-25-8. Recuperado en: http://www.deliberaweb.com/dades/documents/497/1272915541.pdf

Hidalgo, H. H. (2001). Teoría de la intervención del estado en la economía. Recuperado de https://www.gestiopolis. 
com/teoria-de-la-intervencion-del-estado-en-la-economia/

Larraín, F. (2006), "Guatemala: los desafíos del crecimiento", Fondo de Cultura Económica, El Trimestre Económico. Vol. 73, Núm. 291. Julio-septiembre ISSN 2448-718X. https://doi.org/10.20430/ ete.v73i291.329

Loening, L. (2002). "The Impact of Education on Economic Growth in Guatemala", Documento de Trabajo núm. 87, Ibero-America Institute for Economic Research, Georg-August-Universität Göttingen.

Marx, K. (1844), Manuscritos económico-filosóficos. Fondo de Cultura Económica, México. 1975. Recuperado en: https:// www.marxists.org/espanol/m-e/1840s/ manuscritos/man1.htm\#1-2

Matute, A. R. y García, I., (2007). Informe Estadístico de la violencia en Guatemala. Programa de Seguridad Ciudadana y Prevención de la Violencia del Programa de las Naciones Unidad para el DesarroIlo -PNUD- Guatemala, Centroamérica. Editorial Magna Terra. pp. 09 - 70.

Méndez, J. S. (1998). El neoliberalismo en México: ¿éxito o fracaso. Revista de Contaduría y Administración-UNAM (191), 6574. Recuperado en: http: / /www.ejournal. unam.mx/rca/191/RCA19105.pdf

MINEDUC, (2018). Anuario Estadístico; Ministerio de Educación de Guatemala, Años 1992 al 2018. Gobierno de la República de Guatemala. Recuperado en: http:// estadistica.mineduc.gob.gt/Anuario/ home.html\#

Murga, J. M. (2014). Carácter colonial del modelo económico imperante en Guatemala. Asociación Por la Unidad de Nuestra América -AUNA-, Costa Rica. Recuperado en: https://connuestraamerica. blogspot.com/2014/06/caracter-colonial-del-modelo-economico.html
Rodríguez, M. (2015a). Área de educación IPNUSAC. Reflexiones en torno al libro "el capital del siglo XXI" de Thomas Piketty. Revista Análisis de la Realidad Nacional. Universidad de San Carlos de Guatemala. Latindex. En línea. ISSN 2227-9113. Año 4. Edición No. 72. pp. 30-41.

Rodríguez, M., A. (2015b). Universidad y Realidad. Enfoques sobre la sociedad y el Estado guatemalteco. Copilador Olmedo España. Universidad de San Carlos de Guatemala, Guatemala. ISBN: 9789929-709-06-5.

Romero, W. y Ramírez, E. (2018). Las élites económicas y la captura de las instituciones de la política fiscal en Guatemala. Élites, captura del estado y desigualdad. clacso. Con el auspicio de: OXFAM. ISBN: 978-987-722-385-9. Buenos Aires, Argentina. pp. 01-74. Recuperado en: https: / /www.clacso.org.ar/ concursos_convocatorias/Estudios_resultados_clacso_oxfam/Informe_Guatemala_vf.pdf

Smith, A. (1776). La riqueza de las naciones. Oficina de Viuda e Hijos de Santander. Valladolid 1794. Traducida al castellano por Ortiz J. (1794). Recuperado en: https://books.google.com. gt / books?hl=es\&l $r=\& i d=V 4 N x A w A A Q-$ BAJ\&oi=fnd\& $p g=P P 1 \& d q=$ Investiga$\mathrm{ci} \% \mathrm{C} 3 \% \mathrm{~B} 3 \mathrm{n}+$ sobre+la+naturaleza+y+causa+de+las+riquezas+de+las+naciones\%2B\&ots=NIOWxExaq9\&sig=9XUwnyyhAdSO4sBV-XwPCevh6JM\#v=onepage\&q $\mathrm{q}=$ Investigaci $\%$ C $3 \% \mathrm{~B} 3 \mathrm{n} \% 20$ sobre $\% 20$ la\%20naturaleza\%20y $\% 20$ causa $\% 20$ de\%20las\%20riquezas\%20de\%20las\%20 naciones $\% 2 B \& f=$ false

Soto, A. C. (2002). Neoliberalismo y globalización económica. Algunos elementos de análisis para precisar los conceptos. Contaduría y Administración, (205), 1326. 
Spencer, H. (2014). El hombre contra el Estado. INNISFREE. (año de publicación del libro original; 1851) pp. 9 - 141. Recuperado en: https://books.google.es/ books?hl=es\&lr=lang_es\&id=08xjBAAAQBAJ\&oi=fnd\&pg=PA9\&dq=Estaticas+sociales,+o+las+condiciones+esenciales+para+especificar+la+felicidad+humana $+($ Herber + Spenser $)$ \&ots $=k 3 Z b C$ $\mathrm{kHa} 5 \mathrm{y} \& \mathrm{sig}=\mathrm{T} 1 \mathrm{tk0ZnWrg2dZVLutp7Cg}$ $9 q A 80 E \# v=$ onepage\& $q \& f=f a l s e$

Suanes, M., \& Roca, S. O. (2015). Inversión extranjera directa, crecimiento económico y desigualdad en América Latina. El trimestre económico, 82(327), 675706. https://doi.org/10.20430/ete. v82i327. 180

Susanne, G., y Romero, W. (2019). Entre el suelo y el cielo. Radiografía multidimensional de la desigualdad en Guatemala. Oxfam, Guatemala. IDIES/URL.

Wallerstein, I. (2015). ¿El fin de qué modernidad? Revista del Departamento de So- ciología, SOCIOLÓGICA. Año 10. Vol. 27

Sociológica México, (27), 13-31.

\section{SOBRE AUTOR}

\section{Mtro. Marco Antonio Martínez Cuestas}

Es Ingeniero Agrónomo, Maestría en Desarrollo Agropecuario Sustentable de la Universidad de Juarez Autónoma de Tabasco, México y Doctorado en Investigación por la Universidad de San Carlos de Guatemala, Profesor Titular del Centro Universitario de Petén -USAC-, Miembro Propietario de la RED Internacional de Ciencia, Tecnología e Innovación de Guatemala y Revisor de la Revista TERRA LATINOAMERICANA de la Universidad de Chapingo, México.

Conflicto de intereses

Declara no tener ningún conflicto de intereses.

Declaración de consentimiento informado

El estudio se realizó, respetando el Código de ética y buenas prácticas editoriales de publicación.

Derechos de uso

Copyright $\odot 2021$ por Marco Antonio Martínez Cuestas

Este texto está protegido por una licencia Creative Commons 4.0. Internacional

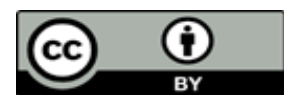

Usted es libre para compartir, copiar y redistribuir el material en cualquier medio o formato y adaptar el documento, remezclar, transformar y crear a partir del material para cualquier propósito, incluso comercialmente, siempre que cumpla la condición de atribución: usted debe reconocer el crédito de una obra de manera adecuada, proporcionar un enlace a la licencia, e indicar si se han realizado cambios. Puede hacerlo en cualquier forma razonable, pero no de forma tal que sugiera que tiene el apoyo del licenciante o lo recibe por el uso que hace. 